\title{
Total elbow arthroplasty for end-stage rheumatoid elbow arthritis with major instability: a case report
}

\author{
Diogo A. Sousa*, Rita Sapage, Carlos Branco, Rita Sousa, Joao Reis, \\ Antonio L. Lopes, Antonio G. Cruz.
}

Department of Orthopaedics, Centro Hospitalar de Trás-os-Montes e Alto Douro, Vila Real, Portugal

Received: 05 June 2020

Revised: 17 July 2020

Accepted: 29 July 2020

*Correspondence:

Dr. Diogo A. Sousa,

E-mail: diogo7sousa@gmail.com

Copyright: () the author(s), publisher and licensee Medip Academy. This is an open-access article distributed under the terms of the Creative Commons Attribution Non-Commercial License, which permits unrestricted non-commercial use, distribution, and reproduction in any medium, provided the original work is properly cited.

\section{ABSTRACT}

A 73 years old female diagnosed with rheumatoid arthritis for over 30 years with an end-stage right elbow arthritis (stage V of Larsen's classification) with a major instability. The patient presented gross deformity and severe pain with critical limitation of limb function. The patient was treated with a total elbow arthroplasty with an intraoperative olecranon fracture. At six months follow up, the patient presents with an excellent active range of motion and painless joint, without daily activities limitation.

Keywords: Rheumatoid arthritis, Elbow instability, Elbow arthritis, Total elbow arthroplasty

\section{INTRODUCTION}

Rheumatoid arthritis (RA) is the most common inflammatory arthropathy in adults and most frequently affects hand and wrist joints. The elbow is also commonly affected (ranging from $20 \%$ to $65 \%$ ). ${ }^{1}$

The elbow motion is essential for the upper limb function, thus pain, instability or stiffness result in an important limitation in patients' daily activities. Despite the impact of disease modifying medications in RA natural history, some patients still present severe elbow degeneration in which the arthroplasty is the best therapeutic option. ${ }^{2,3}$

The classification most commonly used in the rheumatoid elbow is the Larsen's classification which sorts the evolution of the disease in six grades: grade 0 - normal conditions; grade I - slight abnormality; grade II - definite early abnormality; grade III - medium destructive abnormality; grade IV - severe destructive abnormality; grade $\mathrm{V}$ - mutilating abnormality. ${ }^{4}$
We present a case of a patient suffering from a longstanding RA with an end-stage elbow arthritis with major instability and severe pain treated with a total elbow arthroplasty (TEA).

\section{CASE REPORT}

A 73-year-old female diagnosed with RA for over 30 years presents with progressive worsening of pain and range of motion limitation in the right elbow. Of notice, had prior surgical history of bilateral total knee arthroplasty and a right total hip arthroplasty.

At physical examination, the patient presents pain, crepitation and a decreased active range of motion (flexion-extension $45-120^{\circ}$; pronation-supination $10-20^{\circ}$ ) with a multidirectional unstable right elbow. No neurovascular deficits were found. According to the Larsen's classification, the radiographs show a grade $\mathrm{V}$ arthritis with gross bone deformation, destruction of the articular surfaces in the humerus, ulna and radius, as well as displacement in the anteroposterior view. (Figure 1) 
The patient was submitted to a TEA through a lateral paraolecranon approach. As expected, it was found an extensive destruction of the articular surfaces and a major instability.

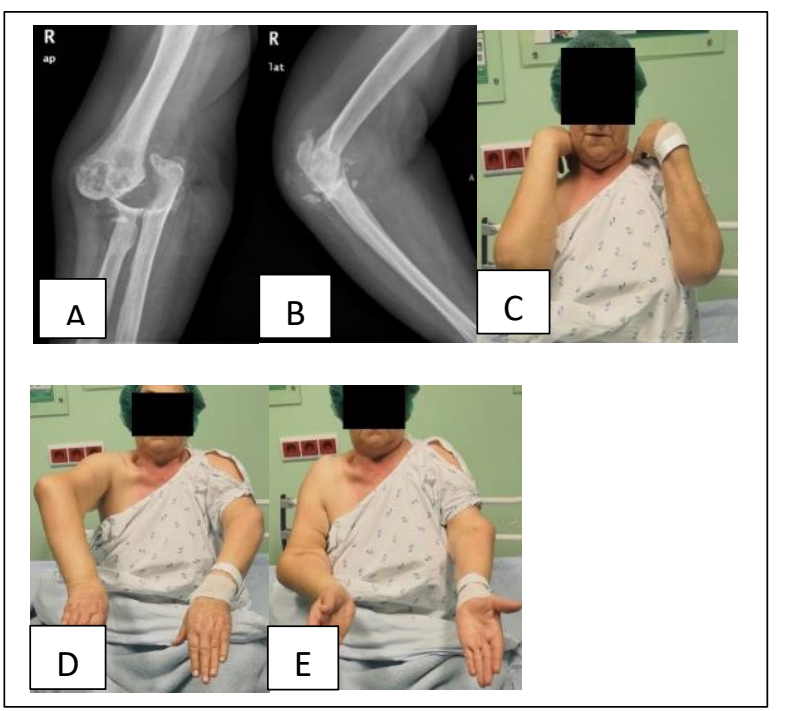

Figure1: Pre-operative images. (A, B) radiographic study of the right elbow (AP- anteroposterior; lat lateral), (C-E) range of motion.

An intra-operative transverse fracture of the olecranon was noticed. As the integrity of the extensor apparatus was maintained, it was repaired with a heavy non-absorbable braided Krackow suture and trans osseous fixation. (Figure 2)

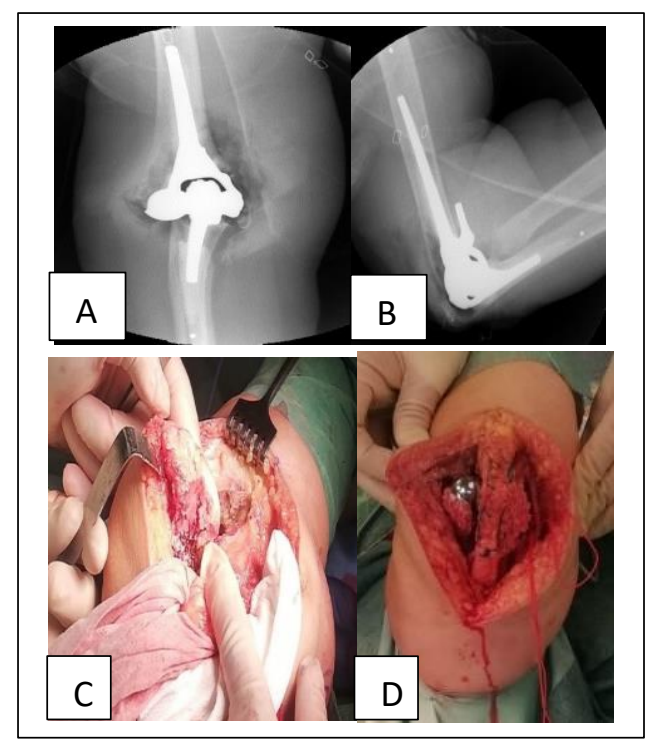

Figure 2: Intra-operative images.

$(\mathrm{A}, \mathrm{B})$ intraoperative imaging with anteroposterior and lateral views, $(C)$ gross bony deformities presented before any bone cut, (D) TEA implanted with the repair of the extensor apparatus and the cubital nerve identified.
The stability and range of motion was checked at the end of the procedure and considered appropriate. In the immediate post-operative, the elbow was immobilized in extension and supination during 4 days, after which initiated the rehabilitation. At six weeks follow up, the patient had no pain and presented a good range of motion (flexion-extension $10-110^{\circ}$; pronation-supination- $70-60^{\circ}$ ) with a stable elbow. The radiographs show a well implanted prosthesis, with a small dislocation of the olecranon fragment. At six months follow up, the patient is painless show an excellent active range of motion (flexion-extension $0-135^{\circ}$; pronation-supination- $80-60^{\circ}$ ). The radiographs present a further dislocation of the olecranon fragment, which to the date as shown no clinical repercussions. (Figure 3)

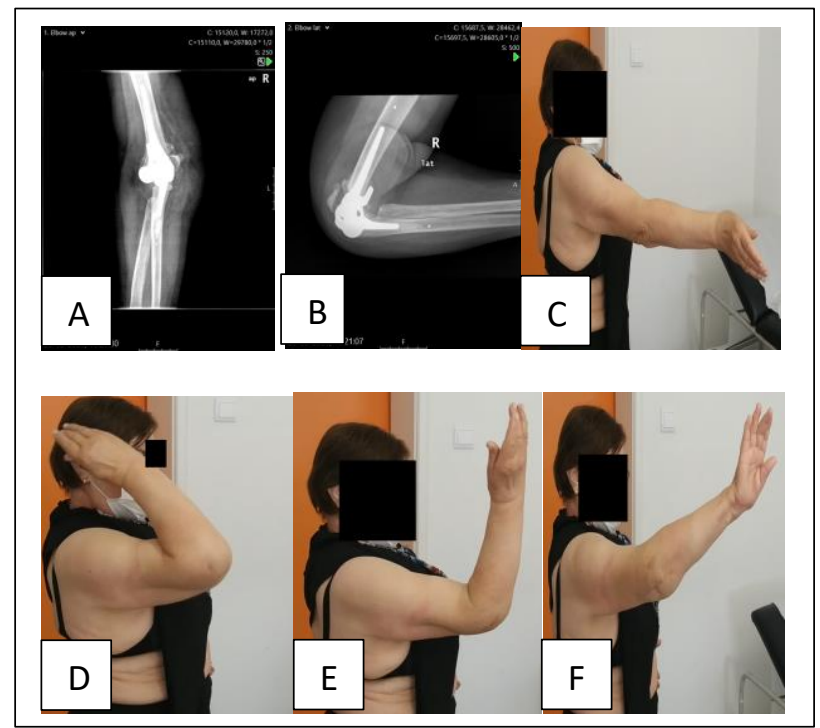

Figure 3: Six month follow up. (A, B) intraoperative imaging with anteroposterior and lateral views. The dislocation of the olecranon fragment can be noticed. (C- F) range of motion.

The patient is very satisfied with the results of the intervention and motivated for the same solution for the contralateral elbow which has a milder form of affection.

\section{DISCUSSION}

In patients with RA the elbow is frequently affected. Despite the impact of disease-modifying antirheumatic drugs, some patients still progress to end-stage elbow arthritis which has a strong negative impact in the quality of life and in the ability to perform daily activities. In fact, these cases represent the most common indication for TEA. $^{5}$

In these cases, the TEA presents the best solution regarding to pain relief and limb function. Although, when compared to other joint replacements such as knee or hip, the TEA is associated with more complications such as infection, aseptic loosening or periprosthetic fractures. ${ }^{6}$ Nishida $\mathrm{K}$ et al reported one patient with a periprosthetic olecranon fracture that was managed conservatively and 
united without relevant clinical implications. ${ }^{7}$ This finding was in accordance with our case.

\section{CONCLUSION}

In patients with symptomatic end-stage rheumatoid elbow, the TEA remains the most favored therapeutic option to restore limb function and providing symptomatic relief. Although it is associated with a high incidence rate of complications, consistent good outcomes and patient satisfaction are achieved with this surgical treatment.

Funding: No funding sources

Conflict of interest: None declared

Ethical approval: Not required

\section{REFERENCES}

1. Studer A, Athwal GS. Rheumatoid arthritis of the elbow. Hand Clin. 2011;27(2):139.

2. Dyer GS, Blazar PE. Rheumatoid elbow. Hand Clin. 2011;27(1):43-8.

3. Nahar K, Nahar N. Management of unusual metallosis and failed elbow replacement in rheumatoid female: a case report. Int J Res Orthop. 2020;6:413-5.
4. Larsen A, Dale K, Eek M. Radiographic evaluation of rheumatoid arthritis and related conditions by standard reference films. Acta Radiol Diagn (Stockh). 1977;18(4):481-91.

5. Kwak JM, Koh KH, Jeon IH. Total Elbow Arthroplasty: Clinical Outcomes, Complications, and Revision Surgery. Clin Orthop Surg. 2019;11(4):369-379.

6. Strelzow JA, Frank T, Chan K, Athwal GS, Faber KJ, King GJW. Management of rheumatoid arthritis of the elbow with a convertible total elbow arthroplasty. J Shoulder Elbow Surg. 2019;28(11):2205-14.

7. Nishida K, Hashizume K, Nasu Y. Mid-term results of alumina ceramic unlinked total elbow arthroplasty with cement fixation for patients with rheumatoid arthritis. Bone Joint J. 2018;100-B(8):1066-73.

Cite this article as: Sousaa DA, Sapagea R, Brancoa C, Sousaa R, Reisa J, Lopesa AL, et al. Total elbow arthroplasty for end-stage rheumatoid elbow arthritis with major instability: a case report Int J Res Orthop. 2020;6:1090-2. 